\title{
Identification of the Low Density Lipoprotein Receptor-binding Site in Apolipoprotein B100 and the Modulation of Its Binding Activity by the Carboxyl Terminus in Familial Defective Apo-B100
}

\author{
Jan Borén, ${ }^{* \ddagger}$ Isabelle Lee, ${ }^{*}$ Weimin Zhu, ${ }^{*}$ Kay Arnold, ${ }^{*}$ Stacy Taylor, ${ }^{*}$ and Thomas L. Innerarity ${ }^{\star \neq \S}$ \\ * Gladstone Institute of Cardiovascular Disease, ${ }^{\ddagger}$ Cardiovascular Research Institute, and ${ }^{\S}$ Department of Pathology, University of \\ California, San Francisco, California 94141-9100
}

\begin{abstract}
Familial defective apolipoprotein B100 (FDB) is caused by a mutation of apo-B100 (R3500Q) that disrupts the receptor binding of low density lipoproteins (LDL), which leads to hypercholesterolemia and premature atherosclerosis. In this study, mutant forms of human apo-B were expressed in transgenic mice, and the resulting human recombinant LDL were purified and tested for their receptor-binding activity. Site-directed mutagenesis and other evidence indicated that Site B (amino acids 3,359-3,369) binds to the LDL receptor and that arginine-3,500 is not directly involved in receptor binding. The carboxyl-terminal $20 \%$ of apo-B100 is necessary for the R3500Q mutation to disrupt receptor binding, since removal of the carboxyl terminus in FDB LDL results in normal receptor-binding activity. Similarly, removal of the carboxyl terminus of apo-B100 on receptor-inactive VLDL dramatically increases apo-B-mediated receptorbinding activity. We propose that the carboxyl terminus normally functions to inhibit the interaction of apo-B100 VLDL with the LDL receptor, but after the conversion of triglyceride-rich VLDL to smaller cholesterol-rich LDL, arginine-3,500 interacts with the carboxyl terminus, permitting normal interaction between LDL and its receptor. Moreover, the loss of arginine at this site destabilizes this interaction, resulting in receptor-binding defective LDL. $(J$. Clin. Invest. 1998. 101:1084-1093.) Key words: lipoproteins • LDL receptor • receptor binding • transgenic mice • familial defective apo-B100
\end{abstract}

\section{Introduction}

The interaction between LDL and the LDL receptor plays a major role in determining plasma cholesterol levels in humans and other mammalian species (1). Apo-B100 is the major protein component of LDL and is responsible for the binding of these lipoproteins to the LDL receptor (2). The relevance of this catabolic pathway is best illustrated by the genetic disor-

Address correspondence to Jan Borén, M.D., Ph.D., Wallenberg Laboratory for Cardiovascular Research, Sahlgrenska University Hospital, S-413 45 Göteborg, Sweden. Phone: 46-31-60-22-10; FAX: 46-31-82-37-62; jan.boren@wlab.wall.gu.se

Received 26 September 1997 and accepted in revised form 30 December 1997

J. Clin. Invest.

(C) The American Society for Clinical Investigation, Inc. 0021-9738/98/03/1084/10 \$2.00

Volume 101, Number 5, March 1998, 1084-1093

http://www.jci.org ders familial hypercholesterolemia $(\mathrm{FH})^{1}$ and familial defective apo-B100 (FDB), in which high levels of LDL accumulate in the circulation because mutations in the LDL receptor $(\mathrm{FH})$ or in the ligand (FDB) disrupt the binding of LDL to its receptor (2). Many different mutations of the LDL receptor cause FH (3), but FDB is associated with a single site mutation, the substitution of glutamine (4), or, in a few cases, tryptophan (5) for the normally occurring arginine at residue 3,500 of apoB100. With the exception of an arginine to cysteine mutation at residue 3,531 (6), which is associated with a minor decrease in LDL receptor binding, extensive searches have not found any other mutations of apo-B100 that cause defective receptor binding of LDL (6). The FDB mutation occurs at an estimated frequency of $1 / 500$ in the normal population and is therefore one of the most common single-gene defects known to cause an inherited abnormality (2).

Much attention has focused on understanding the molecular interaction between apo-B100 and the LDL receptor. The structural and functional domains of the LDL receptor have been defined in detail (3), but much less is understood about the receptor-binding domain of apo-B100, because of its large size and insolubility in aqueous buffer. Furthermore, because apo-B100 binds to the LDL receptor only after the conversion of large VLDL to smaller LDL (1), both its lipid composition and its conformation appear to be crucial to its function as an effective ligand for the LDL receptor,

Although the exact receptor-binding site has remained elusive, several lines of evidence have suggested that residues 3,000-3,700 are critical for mediating apo-B100 binding to the LDL receptor. Milne et al. (7) mapped the epitopes of apo-B monoclonal antibodies that inhibit the binding of LDL to its receptor to a region of apo-B100 in the vicinity of the R3500Q mutation. Another approach involved reacting apo-B100 monoclonal antibodies with LDL that were already bound to the LDL receptor. Those antibodies with epitopes in the region of residues 3,000-3,700 bound poorly, if at all, to the receptorbound LDL (7).

LDL isolated from patients with the genetic disorder hypobetalipoproteinemia have also been useful in defining the apoB100 receptor-binding site. These patients have mutations that result in premature termination of translation and the formation of truncated apo-B. Interestingly, LDL containing the amino-terminal $67 \%$ of apo-B100 (apo-B67) did not bind to the LDL receptor (8). Since apo-B67 contains the first 3,040 amino acids of apo-B100, the receptor-binding domain probably lies carboxyl-terminal to residue 3,040. The LDL with truncated apo-B100 containing the protein's amino-terminal $75 \%$

1. Abbreviations used in this paper: FDB, familial defective apo-B100; $\mathrm{FH}$, familial hypercholesterolemia; RARE, RecA-assisted restriction endonuclease cleavage. 
(apo-B75) or $89 \%$ (apo-B89) bound with an increased affinity to the LDL receptor $(9,10)$. However, these results must be evaluated with caution because SDS-polyacrylamide gels of apo-B77 LDL indicated the presence of apo-E, the other apolipoprotein ligand for the LDL receptor, which may have been responsible for the enhanced binding of this lipoprotein fraction (10).

Selective chemical modification of the apo-B100 of LDL demonstrated that the basic amino acids arginine and lysine were important in the interaction of LDL with its receptor (11, 12). Once apo-B100 was sequenced, several regions enriched in arginine and lysine residues became candidates for receptor binding, including Site A (residues 3,147-3,157) and Site B (residues 3,359-3,367) (13). The receptor-binding site of apo-E has been defined in detail because it is much smaller than apoB100 (299 versus 4,536 residues) and because many different natural mutations of apo-E disrupt receptor binding (14). One of the basic sequences of apo-B100, Site B, is highly similar to the receptor-binding site of apo-E (13).

The current lack of detailed information about the conformation of the apo-B molecule and the mechanism of its interaction with the LDL receptor makes it difficult to understand why only one mutation in apo-B100, the single amino acid substitution in FDB, has such a dramatic effect on receptor binding and why truncated forms of apo-B apparently display increased receptor binding. In this study, we defined the receptor-binding site (Site B) and developed a model that explains how sequences and mutations carboxyl-terminal to the LDL receptor-binding site modulate the receptor-binding activity of apo-B for the LDL receptor. This model explains both the defective receptor binding seen in FDB subjects and the enhanced receptor binding and clearance of truncated forms of apo-B100. It also explains why VLDL do not bind to the LDL receptor and reveals a novel function of the carboxyl terminus of apo-B100. Our results suggest that the carboxyl terminus of apo-B100 inhibits the interaction of VLDL with the LDL receptor and that after conversion of VLDL to smaller LDL, arginine at residue 3,500 stabilizes the carboxyl terminus, permitting normal interaction between LDL and its receptor.

\section{Methods}

Generation of truncated P1 plasmids and isolation of DNA for mutagenesis. The 95-kb apo-B P1 plasmid p158 (15) was prepared and modified by RecA-assisted restriction endonuclease (RARE) cleavage as described (16). Oligomers EcoRI-35,763 and EcoRI-41,496 were used to make a 5.7-kb-deleted P1 plasmid, and oligomers EcoRI39,948 and EcoRI-41,496 to generate a 1.5-kb-deleted P1 plasmid and the corresponding $1.5-\mathrm{kb}$ fragment, respectively (Table I). A 4.2$\mathrm{kb}$ and a $5.7-\mathrm{kb}$ fragment were isolated from the apo-B100 Leu-Leu plasmid with RARE cleavage using oligomers EcoRI-35,763 and EcoRI-39,948 and oligomers EcoRI-35,763 and EcoRI-41,496, respectively. The isolated $1.5-\mathrm{kb}$ fragment and the two fragments with the Leu-Leu mutation were cloned into the pZErO-1 vector. The apo-B100 "Leu-Leu" plasmid was used to increase the yield of apoB100, because it contains a CAA to CTA mutation in codon 2,153 that effectively abolishes the formation of apo-B48, which is formed by an editing mechanism in mouse liver (17).

Generation of mutant $P 1$ clones with mutations at residue 3,500 in apo-B100. The plasmid pZErO-1.5 kb was subjected to site-specific mutagenesis with the Morph System (5 Prime $\rightarrow 3$ Prime, Inc., Boulder, CO). Three different oligomers were used to change arginine at position 3,500 in apo-B to glutamine (R3500Q), glycine (R3500G), or lysine (R3500K) (Table I). After RARE cleavage of the $1.5-\mathrm{kb}-$ deleted P1 plasmid with oligonucleotide EcoRI deleted 1.5-kb, the mutated $1.5-\mathrm{kb}$ fragment was ligated into the linearized and phosphatased 5.7-kb-deleted P1 vector (16). These and all of the following constructs were sequenced over the mutated region(s) and over the 5' and $3^{\prime}$ EcoRI sites and found to be correct.

Generation of mutant $P 1$ clones with apo-B77, apo-B-80, and apo$B 80$ with the $R 3500 Q$ mutation. Oligomers apo-B77 or apo-B80 were used to introduce stop codons at residue 3,497 (apo-B77) or residue 3,620 (apo-B80) in the plasmid pZErO-1.5 kb using the Morph System. The latter mutation was also introduced into the pZErO-1.5 kb $\mathrm{R} 3500 \mathrm{Q}$ plasmid. The mutated $1.5 \mathrm{~kb}$ fragments were isolated by EcoRI digestion and ligated into the vector pZErO-4.2 $\mathrm{kb}$ that had been linearized by RARE cleavage with oligomer EcoRI-39948. The resulting plasmids were subjected to RARE cleavage with oligomers EcoRI-35,763 and EcoRI-41,496, and the mutated 5.7-kb fragments were isolated and ligated into the 5.7-kb-deleted P1 plasmid (16).

Generation of mutant P1 clones with mutations in Site B. The pZErO-5.7-kb plasmid was subjected to site-directed mutagenesis with the ExSite PCR System (Stratagene, Inc., La Jolla, CA). The first mu-

Table I. Oligomers Used in This Study

\begin{tabular}{ll} 
Oligonucleotide & Sequence $\left(5^{\prime}\right.$ to $\left.3^{\prime}\right)$ \\
EcoRI-35763 & GAAAACTCCCACAGCAAGCTAATGATTATCTGAATTCATTCAATTGGGAGAGACAAGTTTCAC \\
EcoRI-39948 & GAACTATTGCTAGTGAGGCCAACACTTACTTGAATTCCAAGAGCACACGGTCTTCAGTGAAGCTG \\
EcoRI-41496 & CACAAGTGAAATATCTGGTTAGGATAGAATCTCCCAGTTTCACAATGAAAACATC \\
EcoRI deleted $1.5 \mathrm{~kb}$ & GAACTATTGCTAGTGAGGCCAACACTTACTTGAATTCTCCCAGTTTTCACAATGAAAACATC \\
EcoRI deleted $4.2 \mathrm{~kb}$ & GAAACTCCCACAGCAAGCTAATGATTATCTGAATTCCAAGAGCACACGGTCTTCAGTGAAG \\
EcoRI deleted $5.7 \mathrm{~kb}$ & GGAAAACTCCCACAGCAAGCTAATGATTATCTGAATTCTCCCAGTTTTCACAATGAAAACATC \\
R3500Q & CCAAGAGCACACAGTCTTCAGTG \\
R3500G & CCAAGAGCACAGGGTCTTCAGTG \\
R3500K & CCAAGAGCACAAAGTCTTCAGTG \\
Apo-B77 & CCGGGCTGCAGGAATTCCTAGAGCACACGG \\
Apo-B80 & GGGTCTTTCCAGAGCTAGGTCGAGCTTTCC \\
Uncharged Site B upper & CTTGACAAGCGCTAGCGGATTGGCGTTAGCCACAGCTCTGTCTCTGAGC \\
Uncharged Site B lower & CTTGTGGTGCCCTCTAATTTGTACTG \\
Apo-E Site B upper & CTGCGCAAGCTGCGTAAGCGGCTCCTCCGCGATGCCGATGCTCTGTCTCTGAGCAACAAATTTGTGG \\
Apo-E Site B lower & GGTGCCCTCTAATTTGTACTGCAGTGC \\
\hline
\end{tabular}


tant was created with oligomers designated uncharged Site-B upper and uncharged Site-B lower. All arginines between residues 3,359 and 3,369 in apo-B were changed to serines, and all the lysines to alanines. The second mutant was created with oligomers designated apo-E Site-B upper and apo-E Site-B lower to substitute residues 3,3583,370 in apo-B with the receptor-binding sequence (residues 141-153) of apo-E. To avoid misincorporation mutations, a 708-nucleotide AvrII-EcoRI fragment of the two vectors, which had been verified by sequencing, was subcloned back into the plasmid pZErO-5.7 kb by RARE cleavage with oligomer EcoRI-39,948 followed by AvrII digestion. The mutated $5.7-\mathrm{kb}$ fragments were isolated and ligated to the linearized 5.7-kb deleted P1 plasmid.

Transgenic mice. Transgenic mice were generated by microinjection of P1 DNA into fertilized mouse eggs (C57BL/6XSJL) as described $(16,18)$. A human apo-B-specific radioimmunoassay of mouse plasma was used to identify transgenic mice (15). Two lines were established from each construct.

Generation of a rabbit antiserum to mouse apo-B and mouse apo-E. Mouse apo-B was isolated from mouse LDL $(d=1.02-1.05 \mathrm{~g} / \mathrm{ml})$. Mouse apo-E was expressed in vitro and isolated. Rabbits were immunized with $5 \mu \mathrm{g}$ of mouse apo-B or $2.5 \mu \mathrm{g}$ of mouse apo-E. The specificity of the antibodies was confirmed by Western analysis. No cross-reactivity against human apo-B or apo-E was detected in the antisera used.

Isolation of recombinant lipoproteins. Blood from mice fasted for $5 \mathrm{~h}$ was collected by cardiac puncture into tubes containing EDTA (final concentration, $1 \mathrm{mg} / \mathrm{ml}$ ), and the plasma was mixed with butylated hydroxytoluene (final concentration, $25 \mu \mathrm{M}$ ), phenylmethyl sulfonylfluoride (final concentration, $1 \mathrm{mM}$ ), and aprotinin (final concentration, $10 \mathrm{U} / \mathrm{ml})$. The LDL $(d=1.02-1.05 \mathrm{~g} / \mathrm{ml})$ were isolated by sequential ultracentrifugation ( $\mathrm{Ti} 70$ rotor) and dialyzed against $150 \mathrm{mM}$ $\mathrm{NaCl}$ and $0.01 \%$ EDTA, pH 7.4, and the mouse apo-E and apo-B were removed by immunoaffinity chromatography. The $d=1.02$ $1.05 \mathrm{~g} / \mathrm{ml}$ fraction was mixed with an equal volume $(850 \mu \mathrm{l})$ of AffiGel Hz (Bio-Rad Laboratories, Richmond, CA) and incubated for $16 \mathrm{~h}$ at $4^{\circ} \mathrm{C}$ in a gently rocking tube filled with $\mathrm{N} 2$. The Affi-Gel $\mathrm{Hz}(100 \mathrm{~g})$

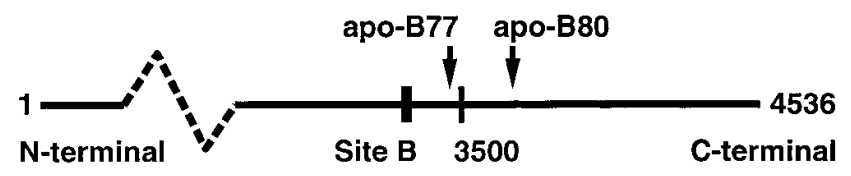

Controls P1-B100

P1-B100 Leu-Leu

3500 Mutations P1-B100 R3500Q (FDB)

P1-B100 R3500K

P1-B100 R3500G

Truncations had previously been coupled with mouse apo-E or mouse apo-B rabbit immunoglobulins from $50 \mathrm{ml}$ of antiserum. Lipoproteins used for receptor-binding experiments were isolated and assayed within $1 \mathrm{wk}$. Human plasma LDL, isolated from a single blood donor, were included as a control in each experiment.

Cell culture and receptor-binding assays. Competitive receptor-binding assays were performed as described (19). The amount of unlabeled lipoproteins needed to compete $50 \%$ with ${ }^{125} \mathrm{I}$-labeled LDL after a 3-h incubation at $4^{\circ} \mathrm{C}$ was determined from an exponential decay curve-fitting model. Direct binding experiments were conducted with increasing concentrations of ${ }^{125}$ I-labeled lipoproteins during a 4-h incubation. Surface-bound radioactivity data were linearized by Scatchard analysis, and the $K_{\mathrm{d}}$ and maximum amount of lipoprotein bound at receptor saturation $\left(B_{\max }\right)$ were determined (19). The results shown are representative of two to four independent receptorbinding assays for each experiment.

Immunoassays of human recombinant $R 3500 Q$ and control $L D L$. Plate immunoassays were carried out as described (20), with the following modifications. The dilution curves of the recombinant $\mathrm{R} 3500 \mathrm{Q}$ and control LDL ranged from $15 \mathrm{ng} / \mu \mathrm{l}$ to $1,500 \mathrm{ng} / \mu \mathrm{l}$. The monoclonal antibodies MB47 and MB3 were diluted 1:10,000 and $1: 15,000$, respectively, and $400,000 \mathrm{cpm}$ of the ${ }^{125}$ I-labeled antibody was added per well.

Analysis of the plasma lipoproteins. Purified lipoproteins were analyzed on Western blots of 3-15\% polyacrylamide-SDS gels, using the enhanced chemiluminescense Western blotting detection reagents (Amersham Corp., Arlington Heights, IL). Negative-staining electron microscopy was performed as described (21), and the particle size distribution in each lipoprotein fraction was determined by measuring at least 1,000 particles with an Image-1/AT analysis system.

\section{Results}

Generation of transgenic mice expressing mutant recombinant human apo-B. Transgenic mice were generated with a human apo-B P1 plasmid in which mutations had been introduced by RARE cleavage. For this study, we created 10 lines of transgenic mice classified into four groups (Fig. 1). The first group consisted of controls. The first control (P1-B100) was human recombinant apo-B100 without any mutation in the apo-B gene. The second control (apo-B100 Leu-Leu) was created to improve the yield of recombinant LDL by eliminating the formation of apo-B48. In the second group, codon 3,500 of the human apo-B gene was altered to understand how mutations affecting residue 3,500 cause defective receptor binding. Residue 3,500 was substituted with either glutamine (R3500Q), glycine (R3500G), or lysine (R3500K). In the third group, truncations were created to localize the receptor-binding site of apo-B100 and to clarify how mutations at residue 3,500 disrupt receptor binding. These deletion mutations were apo-B77 (truncated at residue 3,497), apo-B80 (truncated at residue 3,620), and apoB80 with the FDB mutation (apo-B80 R3500Q). The fourth group was designed to identify the site on apo-B100 that binds to the LDL receptor. Because the basic amino acids arginine

Table II. Mutations in Site B of Apo-B100

\begin{tabular}{lcccr}
\hline Residues & 3359 & & & 3369 \\
Apo-B100 & Arg & Leu Thr Arg Lys Arg Gly Leu Lys Leu Ala \\
Uncharged Site B & Ser & Ser Ala Ser & Ala \\
Apo-E Site B & \multicolumn{2}{c}{ Lys Leu } & Leu & Arg
\end{tabular}

Basic amino acids are in bold.
Figure 1. Generation of transgenic mice. The mutated apo-B P1 clones were used to generate four groups of transgenic mice expressing recombinant LDL containing mutated human apo-B. To improve the yield of the recombinant LDL, all lines in groups three and four and the control apo-B100 Leu-Leu contained the Leu-Leu mutation. A simplified apo-B100 diagram (above) shows the locations of the mutations. 
and lysine are important in receptor binding, the arginine residues were converted to serines and the lysine residues were changed to alanines in Site B of apo-B100 (Table II). Furthermore, because of the homology to the receptor-binding site of apo-E, the receptor-binding region of human apo-E was substituted for site-B of apo-B100.

Isolation, purification, and characterization of recombinant human LDL. Recombinant LDL were isolated from transgenic mouse plasma by ultracentrifugation, and the endogenous mouse apo-E- and apo-B-containing lipoproteins removed by immunoaffinity chromatography. Purified recombinant LDL isolated from the plasma of 10 lines of transgenic mice contained nondegraded apo-B without visible contamination by apo-E or any other protein (Fig. 2). Western blot analysis showed that all recombinant LDL bound to the monoclonal antibody 1D1, whose epitope is between amino acids 474 and 539 in human apo-B100 (22), and that only the unpurified LDL reacted with polyclonal antibodies against mouse apo-B and mouse apo-E (Fig. 3), confirming that mouse apoB100 and apo-E had been completely removed by the immunoaffinity columns. Negative-staining electron microscopy of isolated recombinant LDL showed that the average size of recombinant LDL containing apo-B100 was the same as that of

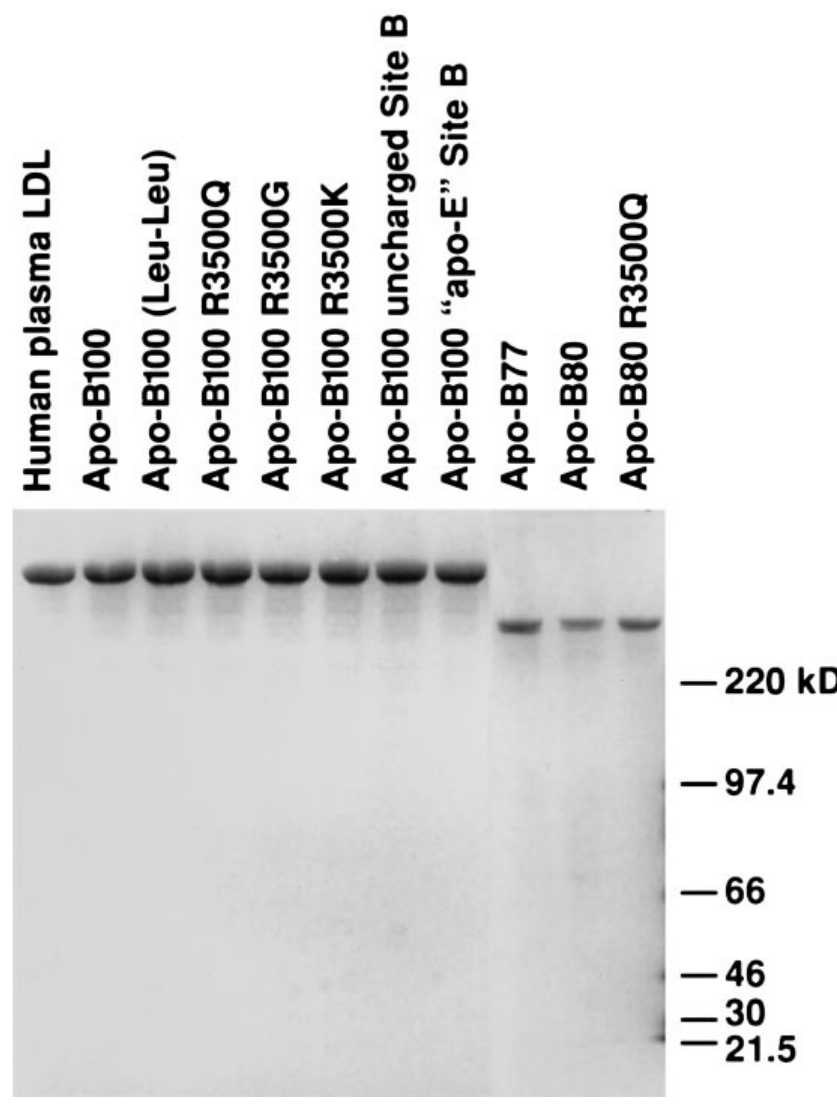

Figure 2. Coomassie staining of recombinant lipoproteins. Recombinant LDL $(d=1.02-1.05 \mathrm{~g} / \mathrm{ml})$ from all transgenic lines were isolated by sequential ultracentrifugation and subjected to immunoaffinity chromatography to remove endogenous apo-B and apo-E. $5 \mu \mathrm{g}$ of apo-B100 (lanes 1-8) and $2 \mu \mathrm{g}$ of the truncated forms of apo-B (lanes $9-11$ ) were analyzed by SDS-PAGE with $3-15 \%$ gels. Human plasma LDL (lane 1) were used as standard.

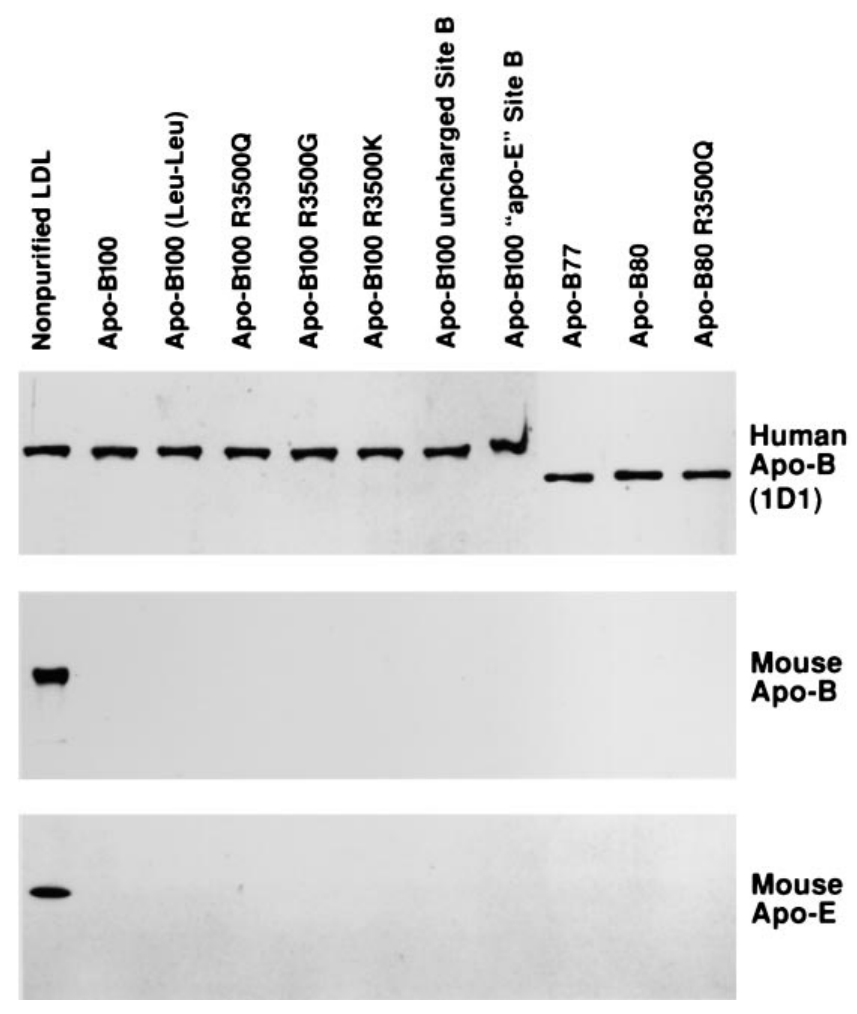

Figure 3. Western analysis of recombinant lipoproteins. $1 \mu \mathrm{g}$ each of nonpurified LDL (lane 1) and purified recombinant LDL (lanes 2-11) were analyzed by Western blots with monoclonal antibody 1D1 against human apo-B (top) and polyclonal antibodies against mouse apo-B (middle) or mouse apo-E (bottom).

human plasma LDL (21.8 2.3 versus $20.8 \pm 1.9 \mathrm{~nm}$, respectively). Recombinant LDL containing truncated forms of apoB100 were slightly smaller (18.8 $\pm 2.1,19.2 \pm 1.9$, and 19.1 \pm 2.4 nm for apo-B77, apo-B80, and apo-B80 R3500Q LDL, respectively). Thus, highly purified recombinant LDL that are structurally similar to plasma-derived human LDL can be isolated from transgenic mice.

Characterization of receptor-binding activity of mousederived human recombinant control LDL. First, we evaluated the receptor-binding activity of purified recombinant LDL derived from the transgenic mouse plasma and found that it was similar to that of plasma-derived human LDL $\left(\mathrm{ED}_{50} 1.9\right.$ versus $1.8 \mu \mathrm{g} / \mathrm{ml}$, respectively). Because the 10 transgenic mouse lines had different transgene copy numbers and different levels of expression of recombinant LDL, we were concerned that the level of expression of the recombinant apo-B might influence the receptor-binding activity of the recombinant LDL. Therefore, we isolated recombinant control LDL from one highexpressor line and one low-expressor line, reflected by the plasma cholesterol concentrations of the pooled blood (229 and $132 \mathrm{mg} / \mathrm{dl}$, respectively). Competitive receptor-binding studies with ${ }^{125} \mathrm{I}-\mathrm{LDL}$ showed almost identical activities $\left(\mathrm{ED}_{50}\right.$ 2.1 versus $2.2 \mu \mathrm{g} / \mathrm{ml}$, respectively). Thus, the receptor-binding affinity was not dependent on the degree of expression of the transgene in the different lines. Nevertheless, two transgenic mouse lines were established from each construct. 
The leu-leu alteration did not influence the receptor binding of the recombinant LDL $\left(\mathrm{ED}_{50} 2.2\right.$ versus $2.3 \mu \mathrm{g} / \mathrm{ml}$, respectively, for the recombinant control LDL with and without this mutation). Thus, we could use recombinant LDL with this mutation, which increased the yield of recombinant LDL.

Verification of the phenotype of the R3500Q mutation. To compare recombinant R3500Q LDL with LDL from an FDB homozygote, we examined the receptor-binding affinities of recombinant R3500Q LDL, LDL from an FDB subject homozygous for the R3500Q mutation, and recombinant control LDL (Fig. $4 A$ ). The $\mathrm{ED}_{50}$ values were 9.8, 10.6, and $2.7 \mu \mathrm{g} / \mathrm{ml}$, respectively. These results were confirmed by direct binding experiments with ${ }^{125}$ I-labeled human plasma-derived LDL, recombinant control LDL, and recombinant R3500Q LDL. Scatchard analysis (Fig. 4 B) showed that the LDL with the R3500Q mutation had a 6.4-fold reduction in affinity for the LDL receptor compared with recombinant control LDL. The $\mathrm{Kd}_{50}$ values for human plasma LDL, recombinant control LDL, or recombinant R3500Q LDL were $0.81,1.31$, and 8.33 $\mu \mathrm{g} / \mathrm{ml}$, respectively. ${ }^{2}$ Thus, both competitive and direct binding experiments demonstrated that the R3500Q mutation causes the defective receptor binding.

LDL from subjects with FDB bind with a higher affinity than control LDL to the apo-B monoclonal antibody MB47. This monoclonal antibody inhibits the binding of LDL to its receptor and has a discontinuous epitope in apo-B100 that flanks residue 3,500 (amino acids 3,429-3,453 and amino acids 3,507-3,523) (23). It has been hypothesized that the R3500Q mutation changes the apo-B100 conformation in this region, such that MB47 binds with a higher affinity to FDB LDL (20). To determine if the R3500Q mutation causes this enhanced MB47 binding we analyzed recombinant LDL with and without the R3500Q mutation for MB47 binding in a solid-phase radioimmunoassay (Fig. 4 C). Less LDL with the R3500Q mutation $\left(\mathrm{ED}_{50} 285 \mathrm{ng}\right)$ was required than recombinant control LDL $\left(\mathrm{ED}_{50} 386 \mathrm{ng}\right.$ ) to displace $50 \%$ of human plasma-derived LDL from MB47. This experiment were repeated three times with newly isolated recombinant LDL for each experiment. These experiments showed that MB47 had a 1.5-fold higher affinity for recombinant R3500Q LDL than for recombinant control LDL (average of $n=3$ ). Taken together, these results prove that the R3500Q mutation enhances MB47 binding and causes the defective receptor binding seen in FDB LDL.

Analysis of human recombinant LDL with mutations at residue 3500. Next, we determined whether the loss of arginine or the loss of a positive charge at residue causes receptor binding-defective LDL. Recombinant LDL with the R3500Q, $\mathrm{R} 3500 \mathrm{~K}$, or R3500G mutation were analyzed in a competitive in vitro receptor-binding assay (Fig. $5 A$ ). Surprisingly, recom-

2. The direct binding experiment showed that recombinant control LDL had slightly lower affinity for the LDL receptor than human plasma LDL. The reason for this is unclear but may be due to differences in lipid composition. Recombinant human or mouse LDL overexpressed in transgenic mice have been shown to be more triglyceride rich than normal human or mouse plasma $\operatorname{LDL}(15,31)$, and increased triglyceride content of the core of the LDL particle has been shown to alter the interaction with the $\operatorname{LDL}$ receptor $(32,33)$. Thus, it is conceivable that the slightly lower LDL receptor affinity is caused by changes in lipid composition.

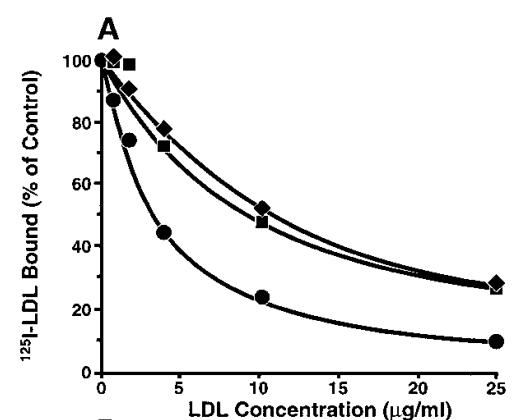

Figure 4. Characterization of recombinant LDL with the R3500Q mutation. (A) The abilities of recombinant R3500Q LDL (squares), receptor binding-defective LDL from a patient with homozygous FDB (diamonds), and recombinant control LDL (circles) to compete with ${ }^{125}$ I-labeled human plasma LDL $(2 \mu \mathrm{g} / \mathrm{ml})$ for binding to LDL receptors on normal human fibroblasts were determined. The recombinant lipoproteins were isolated from 10 mice, and endogenous apo-E and apo-B were removed. Competitor LDL were added at the indicated concentrations to normal human fibroblasts, and the amount of ${ }^{125}$ I-LDL bound to the fibroblasts was measured after a 3-h incubation. $(B)$ Scatchard analysis of the direct binding of ${ }^{125}$ I-LDL from human plasma (circles), recombinant control LDL (triangles), or recombinant R3500Q LDL (squares). The recombinant LDL were isolated from 35 mice each and labeled with ${ }^{125} \mathrm{I}$ after removal of endogenous apo-E and apo-B. Nonspecific binding, obtained by the addition of a 100-fold excess of unlabeled plasma LDL, was subtracted at each concentration.

Bound/Free $(B / F)$ is the amount of LDL bound/dish divided by the amount of LDL free in the medium in each dish. The molar $K_{\mathrm{d}}$ values were $1.35,2.18$, and $13.9 \times 10^{-9}$, respectively. $(C)$ The abilities of recombinant control LDL (circles) and recombinant R3500Q LDL (squares) to bind to MB47 monoclonal antibody. The recombinant LDL were isolated from 10 mice each and analyzed in a solid-phase competitive radioimmunoassay after removal of endogenous apo-E and apo-B.

binant LDL with the R3500K mutation were as defective as the LDL with the R3500Q or the R3500G mutation $\left(\mathrm{ED}_{50}>\right.$ $25 \mu \mathrm{g} / \mathrm{ml}$ versus $5.5 \mu \mathrm{g} / \mathrm{ml}$ for recombinant control apo-B100 LDL). Thus, it is the presence of an arginine at residue 3,500, not simply a positive charge, that is essential for normal receptor binding.

Competitive receptor binding of $L D L$ and $V L D L$ with truncated forms of apo-B. Analysis of truncated LDL isolated from patients with familial hypobetalipoproteinemia showed that apo-B67, containing the first 3,040 amino acids of apo$\mathrm{B} 100$, did not bind to the LDL receptor (8). However, LDL containing either apo-B75, which terminates at residue 3,386, or apo-B89 apparently bound more avidly than normal LDL to the LDL receptor $(9,10)$. How could these truncated forms of apo-B display higher affinity for the LDL receptor than 

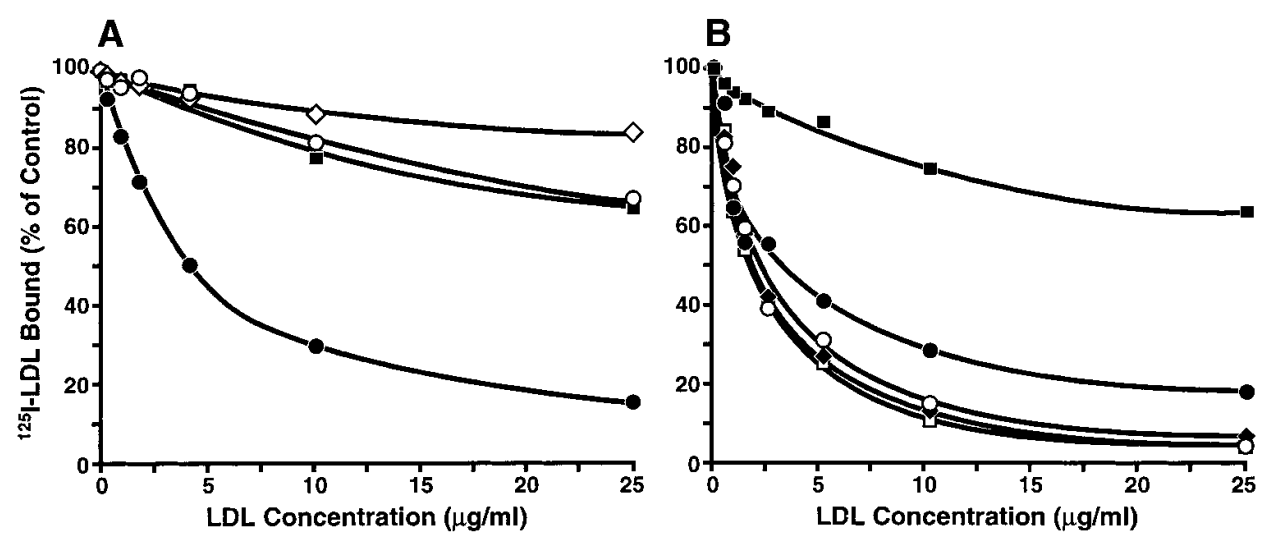

Figure 5. The abilities of different mouse-derived recombinant LDL and VLDL to compete with

${ }^{125}$ I-labeled human plasma LDL for receptor-binding to human fibroblasts. (A) Recombinant control LDL (black circles), recombinant R3500Q LDL (black squares), R3500G LDL (open diamonds), and R3500K LDL (open circles) were isolated from 10 mice each as described and tested for receptor binding. (B) Recombinant control LDL (black circles), recombinant R3500Q LDL (black squares), apoB77 LDL (open squares), apo-B80
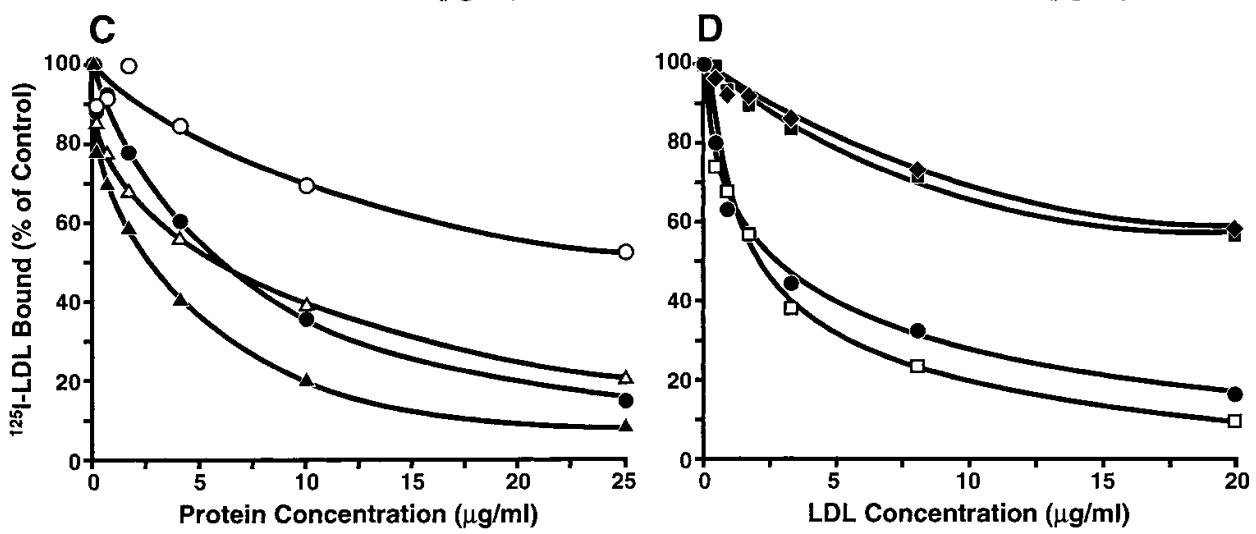

(black diamonds), and apo-B80 R3500Q LDL (open circles) were isolated from 15 mice each as described and tested for receptor binding. $(C)$ Recombinant control LDL (black circles) and VLDL (open circles), and recombinant apo-B80 LDL (black triangles) and VLDL (open triangles) were isolated from 55 control mice and 70 apo-B80 mice, both bred on apo-E-deficient background. $(D)$ Recombinant control LDL (black circles), R3500Q LDL (black squares), LDL with uncharged Site B (black diamonds), and LDL in which Site B was re-

placed with the receptor-binding site of apo-E (open squares) were isolated from 15 mice each. Endogenous apo-B was removed by immunoaffinity chromatography before the recombinant lipoproteins were tested in an in vitro competitive receptor-binding assay. Human plasma LDL was included as a control in each experiments. The $\mathrm{ED}_{50}$ values were $2.5,2.2,2.2$, and $2.6 \mu \mathrm{g} / \mathrm{ml}$, respectively.

apo-B100? One explanation would be an increased apo-E/ apo-B molar ratio in truncated forms of LDL, enhancing the affinity of the apo-B75 LDL for the LDL receptor. This possibility cannot be ruled out, as SDS-polyacrylamide gels of the apo-B75 LDL demonstrated the presence of apo-E, which may have been responsible for the enhanced binding of apoB75 (10). A more intriguing hypothesis is that the carboxylterminal part of apo-B100 itself modulates the receptor binding of LDL.

To test this hypothesis, we isolated and analyzed recombinant LDL containing apo-B77, apo-B80, apo-B80 R3500Q, apo-B100 R3500Q, and recombinant control LDL. Western analysis demonstrated that immunoaffinity purification removed all endogenous apo-B and apo-E (Fig. 1). Competitive receptor-binding assays showed repeatedly that, compared with recombinant control LDL, all LDL containing truncated forms of apo-B had slightly enhanced binding to LDL receptors (Fig. $5 \mathrm{~B}$ ). The $\mathrm{ED}_{50}$ values for recombinant apo-B77, apo-B80, apo-B80 R3500Q, control apo-B100 LDL, and apoB100 R3500Q LDL were 1.9, 1.9, 1.5, 3.7, and $>25 \mu \mathrm{g} / \mathrm{ml}$, respectively. The fact that apo-B77 had increased receptor binding proves that arginine-3,500 is not directly involved in receptor binding: apo-B77 is truncated at residue 3,497 and therefore does not contain arginine-3,500. Moreover, the finding that apo-B80 LDL with the R3500Q mutation has enhanced receptor binding indicates that glutamine at residue
3,500 of full-length FDB LDL interacts with residues in the carboxyl-terminal $20 \%$ of apo-B100 to disrupt receptor binding or, alternatively, that arginine-3,500 in normal LDL interacts with residue(s) in the carboxyl tail to facilitate normal receptor binding.

The observation that the carboxyl terminus of apo-B100 could inhibit LDL binding suggested that this modulation may be important in other apo-B-containing lipoproteins. For example, large VLDL bind poorly to the LDL receptor via apo-B. However, when these large particles are reduced in size by lipolysis, the apo-B100 in the smaller LDL bind with high affinity to LDL receptors (1). To determine if the carboxyl terminus of apo-B100 inhibits the binding of VLDL to LDL receptors, we analyzed the receptor binding of VLDL containing apo-B80 or apo-B100. In these experiments, it was essential not to have any apo-E that would interfere with the receptorbinding assay. Because apo-E is an integral component of VLDL and cannot be removed by immunoaffinity chromatography, transgenic mice expressing recombinant apo-B80 or apo-B100 were bred with gene-targeted apo-E-deficient mice. Furthermore, since endogenous apo-B48 accumulates in apo$\mathrm{E}$-deficient mice, making complete removal of mouse apo-B difficult, the apo-B80 $\times$ apo- $\mathrm{E}^{-1-}$ and apo-B100 $\times$ apo- $\mathrm{E}^{-/-}$ mice were bred onto an endogenous apo-B100-only mouse background. Apo-B100-only mice have been genetically modified to produce only apo-B100 and no apo-B48 (24). VLDL 
$(d<1.006 \mathrm{~g} / \mathrm{ml})$ and LDL $(d=1.02-1.05 \mathrm{~g} / \mathrm{ml})$ were isolated by ultracentrifugation, and the mouse apo-B100 was removed by immunoaffinity chromatography. Negative-staining electron microscopy of the isolated lipoprotein particles showed that the apo-B100 and apo-B80 VLDL, which were the same size ( 35.5 and $34.9 \mathrm{~nm}$, respectively), were substantially larger in diameter than the apo-B100 and apo-B80 LDL (23.5 and 21.5 $\mathrm{nm}$, respectively). Further characterization of the apo-B80 and apo-B100 VLDL showed they had the same lipid composition (data not shown). In competitive binding studies with ${ }^{125}$ I-labeled normal LDL (Fig. 5 C), apo-B80 VLDL bound with a 6.5-fold higher affinity to human fibroblasts than apo-B100 VLDL $\left(E_{50} 6.0\right.$ versus $39 \mu \mathrm{g} / \mathrm{ml}$, respectively). The $\mathrm{ED}_{50}$ for apoB100 VLDL was determined using Probit transformation (19). Apo-B80 LDL bound 2.3-fold more avidly to the LDL receptor than apo-B100 LDL $\left(\mathrm{ED}_{50} 2.6 \mu \mathrm{g} / \mathrm{ml}\right.$ versus $6.0 \mu \mathrm{g} / \mathrm{ml}$, respectively). These results show that the carboxyl-terminal $20 \%$ of apo-B100 inhibits the receptor binding of VLDL and confirm that apo-B80 LDL bind more avidly than apo-B100 LDL.

The receptor-binding site of apo-B100. Studies of LDL from patients with hypobetalipoproteinemia have indicated that the receptor-binding region of apo-B100 probably lies between amino acids 3,040 and 3,386. Analysis of the amino acid sequence of apo-B100 in this domain shows two regions containing clusters of basic amino acids, Site A (residues 3,147-3,157) and Site B (residues 3,359-3,367), which have been proposed as the receptor-binding regions of apo-B100 (13). To identify conserved, functionally significant regions in apo-B100, we analyzed LDL ( $d=1.02-1.05 \mathrm{~g} / \mathrm{ml})$ isolated from Xenopus laevis and made the surprising discovery that $X$. laevis LDL bind to human LDL receptors with an affinity equivalent to or higher than that of human LDL (data not shown). Subsequently, we sequenced $4.5 \mathrm{~kb}$ of $X$. laevis apo-B DNA and compared this with Site A and Site B of human apo-B100 (Table III). Site B was essentially perfectly conserved despite 350 million years of evolution, strongly implicating its importance in receptor binding. The fact that Site B is very similar to the receptor-binding site of apo-E also argues in favor of its significance. Moreover, Site A is poorly conserved in both chicken and frog apo-B (Table III), and receptor-binding analysis of chicken LDL $(d=$ $1.02-1.05 \mathrm{~g} / \mathrm{ml}$ ) showed that it binds with a high affinity equivalent to that of human LDL (data not shown). The lack of conservation of Site A in these animals (Table III) indicated that Site A is very unlikely to be a potential site for receptor binding.
Therefore, we focused on Site B. First we changed the basic amino acids in this region to neutral amino acids (Table II), substituting serines for arginines and alanines for lysines. Second, we reasoned that if Site B in apo-B100 actually interacts with the LDL receptor, then it might be possible to substitute the receptor-binding site apo-E for Site B and retain LDL receptor-binding activity. The receptor-binding ability of recombinant LDL with the uncharged Site B or with the receptorbinding site of apo-E substituted for Site B was compared with those of recombinant control LDL and recombinant R3500Q LDL (Fig. 5 D). LDL containing the uncharged Site B had defective receptor binding equivalent to that of recombinant R3500Q LDL $\left(E_{50}>20 \mu \mathrm{g} / \mathrm{ml}\right.$ for both). Furthermore, the LDL with the receptor-binding region of apo-E in place of Site $\mathrm{B}$ and recombinant control LDL had similar receptor-binding activities $\left(\mathrm{ED}_{50} 2.4\right.$ and $2.3 \mu \mathrm{g} / \mathrm{ml}$, respectively). These results prove that Site B is functionally important for LDL receptor binding.

One concern with using site-directed mutagenesis to study protein function is distinguishing between mutations that affect local structure and function and those that affect the folding and stability of the entire protein, because both would have the same defective binding phenotype. To determine if the mutation(s) affected only the structure and function of the receptor-binding site or if they had a more global effect, we used primary mouse hepatocytes to test the rate of secretion of recombinant apo-B from all transgenic lines in vitro. No differences were found (data not shown). The assembly of VLDL and LDL is a complex procedure, and if the protein is not folding correctly, the apo-B100 probably will be degraded instead of being secreted (25).

\section{Discussion}

Even though the apo-B cDNA and gene were cloned and sequenced more than a decade ago, progress in understanding the molecular interaction between apo-B100 and the LDL receptor has been slow. There are several reasons for the slow progress. Apo-B100, one of the largest proteins known $(4,536$ amino acids), is highly hydrophobic and aggregates when not associated with lipids. Thus, only apo-B100 present in LDL can bind to the LDL receptor. Moreover, recombinant LDL secreted by cultured hepatic cells transfected with apo-B minigenes lacked normal receptor-binding activity (Innerarity,

Table III. Species Comparison of Site A and Site B in Apo-B100 with the Receptor-binding Site of Apo-E

\begin{tabular}{|c|c|c|c|c|c|c|c|c|c|c|c|}
\hline \multicolumn{12}{|l|}{ Site A } \\
\hline Apo-B residue & 3147 & & & & & & & & & & 3157 \\
\hline Human & Lys & Ala & Gln & Tyr & Lys & Lys & Asn & Lys & His & Arg & His \\
\hline Chicken & Asn & & & & Glu & & & & Asp & Met & \\
\hline Frog & & Leu & & & & & & Thr & Asp & Val & \\
\hline \multicolumn{12}{|l|}{ Site $B$} \\
\hline Apo-B residue & 3359 & & & & & & & & & & 3369 \\
\hline Human & Arg & Leu & Thr & Arg & Lys & Arg & Gly & Leu & Lys & Leu & Ala \\
\hline Chicken & Ser & & & & & & & & & & \\
\hline Frog & Gly & & & & & & & & & & \\
\hline Apo-E3 residue & 142 & & & & & & & & & & 152 \\
\hline Human & Arg & Lys & Leu & Arg & Lys & Arg & Leu & Leu & Arg & Asp & Ala \\
\hline
\end{tabular}

Basic amino acids are in bold. 
T.L., unpublished observation), and heterologous minigenes yield extremely poor expression in transgenic mice (26). To overcome these problems, we developed a new approach for studying apo-B structure: mutating a P1 bacteriophage clone, making transgenic mice, and then purifying and characterizing the mutant apo-B protein. The first step was to mutate the 95$\mathrm{kb} \mathrm{P} 1$ clone. For this purpose, we developed an efficient protocol for performing site-directed mutagenesis on large-insert bacterial clones based on RARE cleavage, which made it possible to insert subtle or complex mutations into the apo-B gene within $2 \mathrm{wk}$ (16). This strategy made it feasible to generate a series of apo-B mutants to dissect the molecular interaction between apo-B100 and the LDL receptor.

Our study indicates that Site B (residues 3,359-3,369) is critical for receptor binding. Further support for this finding can be summarized as follows: LDL with apo-B truncated at amino acid 3,040 do not bind to the LDL receptor (8), but LDL with apo-B truncated at residue 3,386 (10) or 3,497 bind avidly to the LDL receptor. Site B is within the receptor-binding region defined by these truncated apo-B LDLs. Furthermore, Site B has clusters of basic amino acids known to be important for binding to the LDL receptor and is extremely similar to the site on apo-E that binds to the LDL receptor (13, 14). Site B is highly conserved among many species (27) and essentially perfectly conserved in apo-B100 from $X$. laevis. This conservation over 350 million years of evolution is consistent with Site B's functional importance in receptor binding. The finding that Site B peptides from apo-B100 bind heparin (28) is significant because the receptor-binding site of apo-E also binds heparin. Immunoelectron microscopy studies by Phillips et al. also support this concept (Phillips, M., and V. Schumaker, personal communication). They found that a soluble fragment of the LDL receptor bound immediately aminoterminal to the binding site of the monoclonal antibody MB47 on apo-B100. Since the discontinuous MB47 epitope of apoB100 includes amino acids 3,429-3,453 and 3,507-3,523 (23), these results place the location of binding in the vicinity of Site B (3,359-3,369). Furthermore, our receptor-binding analysis of recombinant LDL with an altered Site B indicated that Site B is functionally important for the interaction between apo-B100 and the LDL receptor. Finally, recombinant LDL maintained normal receptor binding when Site B was replaced with the receptor-binding site of apo-E, indicating that Site B is equiva- lent to apo-E's receptor-binding site and therefore is most likely the site that binds to the LDL receptor. While it has been proposed that the receptor-binding domain is discontinuous and composed of several segments of apo-B100 and that Site B is only one site of several (27), our data argue against this concept. Acetylation of the remaining lysine residues of apo-B100 in the recombinant LDL with the mutated Site B did not further decrease the receptor binding (data not shown). While other interpretations are possible, it is likely that Site B is the primary or only receptor-binding site for apo-B100.

The results from this study together with the elegant mapping of apo-B100 on the LDL particle (29) now allow a broad understanding of the receptor-binding site of apo-B100 and explain how sequences and mutations distal to the receptorbinding region can modulate the affinity of apo-B100 for the LDL receptor. Based on this understanding, we developed the model illustrated in Fig. 6. Chatterton et al. (29) showed that the first $89 \%$ of apo-B100 enwraps the LDL particle like a belt and that the carboxyl-terminal $11 \%$ constitutes a bow that crosses over the belt and brings the carboxyl tail of apo-B100 close to amino acid residue 3,500 . We propose that a region of apo-B100 carboxyl-terminal to Site B and encompassing residue 3,500 serves as a modulator that can alter the affinity of the receptor-binding domain of apo-B100. Moreover, our results suggest that arginine-3,500 interacts with the carboxyl tail of apo-B100 and that this interaction is important for receptor binding of LDL. In our model, this modulator element, or more specifically arginine-3,500, interacts with the carboxyl tail that crosses over at or very close to residue 3,500. The substitution of glutamine for arginine at residue 3,500 (i.e., site R3500Q in Fig. 6) abolishes this interaction, resulting in a conformational change, disrupted receptor binding, and the clinical disorder FDB. Truncation mutations in which there is insufficient carboxyl tail to cross over near Site B do not need this interaction and therefore bind normally without arginine3,500. This explains why LDL containing apo-B80 with the R3500Q mutation have normal or enhanced LDL receptorbinding activity (Fig. 6). Evidence that the R3500Q mutation significantly alters the microenvironment in the region around residue 3,500 of apo-B100 comes from both the MB47 studies showing enhanced binding to FDB LDL (20) and from ${ }^{13} \mathrm{C}$ nuclear magnetic resonance analysis of FDB LDL (30).

Our receptor-binding studies with different types of muta-
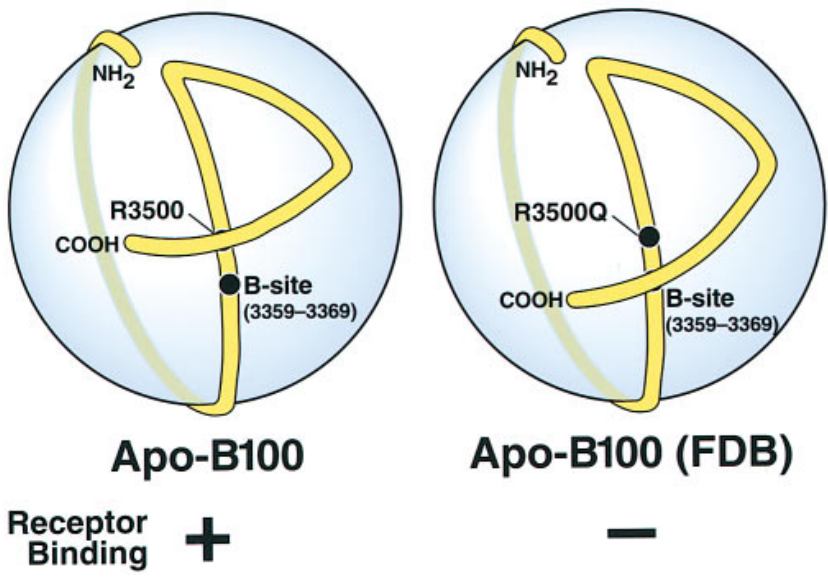

Figure 6. Schematic of the carboxyltail modulator model for apo-B100 binding to the LDL receptor. The first $89 \%$ of apo-B100 enwraps the LDL particle like a belt, and the carboxyl-terminal $11 \%$ constitutes a bow that crosses over the belt, bringing the carboxyl-terminal portion of apo-B100 close to amino acid 3500. The model proposes that apo$\mathrm{B} 100$ binds to the LDL receptor via Site B (residues 3359-3369) and that the bow serves as a modulator element that can alter the affinity of the receptor-binding domain of apoB100. The projection of apo-B100 on the back of the LDL particle has been simplified. 
tions at residue 3,500 demonstrate that it is arginine and not a positive charge at this position that is critical for receptor-binding activity. The importance of arginine at this position is also indicated by the finding that arginine at residue 3,500 is conserved in all species that have been sequenced, including the frog $X$. laevis. The case of the frog is especially interesting because synonymous codons are used for the frog and human apo-B genes to code for arginine-3,500. The finding that lysine cannot substitute for arginine at residue 3,500 suggests that arginine-3,500 does not form a salt bridge to an acidic residue but the nature of this interaction and the sequence(s) arginine3,500 interacts with is an area we are investigating currently.

Our model also proposes that the carboxyl-terminal sequence of apo-B100 functions as a negative modulator of receptor binding and inhibits binding of VLDL to the LDL receptors. We propose that during lipolysis of large VLDL to smaller LDL, the carboxyl terminus changes its conformation to allow interaction with the LDL receptor. Removal of the tail increases not only the receptor binding of VLDL more than sixfold but also, to a lesser extent, the binding of LDL to the LDL receptor. Interestingly, Milne and his colleagues have found that the only dramatic difference in the binding of monoclonal antibodies to apo-B100 in VLDL and LDL occurs with antibodies binding to the carboxyl terminus of the protein (Wang, X., and R. Milne, personal communication). Furthermore, Parhofer et al. (9) demonstrated enhanced clearance of both LDL and VLDL in a subject with apo-B89, indicating that our in vitro observations are most likely physiologically relevant. Our model further proposes, albeit more speculatively, that the hydrolysis of VLDL permits arginine-3,500 to interact with the carboxyl tail, opening up Site B for receptor binding.

In addition to revealing the molecular basis for the genetic disorder FDB, our model explains the increased clearance of certain truncated forms of apo-B found in hypobetalipoproteinemic patients. Our results show that truncation of the apo-B molecule enhanced its affinity for the LDL receptor and that apo-B80 VLDL had an affinity for the LDL receptor similar to that of apo-B100 LDL. The latter finding suggests that VLDL with truncated forms of apo-B containing Site B may bind to the LDL receptor without metabolic processing to LDL and explains the increased clearance of apo-B89 VLDL described by Parhofer et al. (9). Furthermore, the increased clearance of VLDL via the LDL receptor leads to a decreased formation of LDL. This mechanism explains why the plasma lipoprotein distribution in some hypobetalipoproteinemic patients is characterized by an almost complete lack of LDL.

The results from this study now allow a broad understanding of the receptor-binding site of apo-B100 and explain how sequences and mutations distal to the receptor-binding region can modulate the affinity of apo-B100 for the LDL receptor. We suspect that the strategy applied in these studies will be useful for elucidating a wide range of apo-B structure and function issues.

\section{Acknowledgments}

The authors thank Dr. John Kane, Dr. Ross Milne, Dr. Robert W. Mahley, Dr. Martin Phillips, Dr. Stanley C. Rall, Dr. Verne Schumaker, and Dr. Karl Weisgraber for stimulating discussions and comments on the manuscript, Dr. Stephen G. Young for generously providing ${ }^{125}$ I-labeled C1.4 antibodies and for apo-B100 only mice, Dr.
Li-Ming Dong for generous help in expressing and purifying mouse apo-E, S. Ordway and G. Howard for editorial assistance, J. Carrol and B. Clark for help in preparation of the figures, and A. Corder and S. Gonzales for photographic work.

J. Borén is supported by the Howard Hughes Medical Institute with a Howard Hughes Postdoctoral Fellowship for Physicians. This work also was supported by National Heart, Lung, and Blood Institute grant HL-47660.

\section{References}

1. Goldstein, J.L., M.S. Brown, R.G.W. Anderson, D.W. Russell, and W.J. Schneider. 1985. Receptor-mediated endocytosis: concepts emerging from the LDL receptor system. Annu. Rev. Cell Biol. 1:1-39.

2. Innerarity, T.L., R.W. Mahley, K.H. Weisgraber, T.P. Bersot, R.M. Krauss, G.L. Vega, S.M. Grundy, W. Friedl, J. Davignon, and B.J. McCarthy. 1990. Familial defective apolipoprotein B100: a mutation of apolipoprotein B that causes hypercholesterolemia. J. Lipid Res. 31:1337-1349.

3. Hobbs, H.H., M.S. Brown, and J.L. Goldstein. 1992. Molecular genetics of the LDL receptor gene in familial hypercholesterolemia. Hum. Mutat. 1: 445466 .

4. Innerarity, T.L., K.H. Weisgraber, K.S. Arnold, R.W. Mahley, R.M. Krauss, G.L. Vega, and S.M. Grundy. 1987. Familial defective apolipoprotein B-100: low density lipoproteins with abnormal receptor binding. Proc. Natl. Acad. Sci. USA. 84:6919-6923.

5. Gaffney, D., J.M. Reid, I.M. Cameron, K. Vass, M.J. Caslake, J. Shepherd, and C.J. Packard. 1995. Independent mutations at codon 3500 of the apolipoprotein B gene are associated with hyperlipidemia. Arterioscler. Thromb. Vasc. Biol. 15:1025-1029.

6. Pullinger, C.R., L.K. Hennessy, J.E. Chatterton, W. Liu, J.A. Love, C.M. Mendel, P.H. Frost, M.J. Malloy, V.N. Schumaker, and J.P. Kane. 1995. Familial ligand-defective apolipoprotein B. Identification of a new mutation that decreases LDL receptor binding affinity. J. Clin. Invest. 95:1225-1234.

7. Milne, R., R. Théolis, Jr., R. Maurice, R.J. Pease, P.K. Weech, E. Rassart, J.-C. Fruchart, J. Scott, and Y.L. Marcel. 1989. The use of monoclonal antibodies to localize the low density lipoprotein receptor-binding domain of apolipoprotein B. J. Biol. Chem. 264:19754-19760.

8. Welty, F.K., L. Seman, and F.T. Yen. 1995. Purification of the apolipoprotein B-67-containing low density lipoprotein particle and its affinity for the low density lipoprotein receptor. J. Lipid Res. 36:2622-2629.

9. Parhofer, K.G., A. Daugherty, M. Kinoshita, and G. Schonfeld. 1990. Enhanced clearance from plasma of low density lipoproteins containing a truncated apolipoprotein, apoB-89. J. Lipid Res. 31:2001-2007.

10. Krul, E.S., K.G. Parhofer, P.H.R. Barrett, R.D. Wagner, and G. Schonfeld. 1992. ApoB-75, a truncation of apolipoprotein B associated with familial hypobetalipoproteinemia: genetic and kinetic studies. J. Lipid Res. 33:10371050 .

11. Mahley, R.W., T.L. Innerarity, R.E. Pitas, K.H. Weisgraber, J.H. Brown, and E. Gross. 1977. Inhibition of lipoprotein binding to cell surface receptors of fibroblasts following selective modification of arginyl residues in arginine-rich and B apoproteins. J. Biol. Chem. 252:7279-7287.

12. Weisgraber, K.H., T.L. Innerarity, and R.W. Mahley. 1978. Role of the lysine residues of plasma lipoproteins in high affinity binding to cell surface receptors on human fibroblasts. J. Biol. Chem. 253:9053-9062.

13. Knott, T.J., S.C. Rall, Jr., T.L. Innerarity, S.F. Jacobson, M.S. Urdea, B. Levy-Wilson, L.M. Powell, R.J. Pease, R. Eddy, H. Nakai, et al. 1985. Human apolipoprotein B: structure of carboxyl-terminal domains, sites of gene expression, and chromosomal localization. Science. 230:37-43.

14. Mahley, R.W. 1988. Apolipoprotein E: cholesterol transport protein with expanding role in cell biology. Science. 240:622-630.

15. Linton, M.F., R.V. Farese, Jr., G. Chiesa, D.S. Grass, P. Chin, R.E. Hammer, H.H. Hobbs, and S.G. Young. 1993. Transgenic mice expressing high plasma concentrations of human apolipoprotein B100 and lipoprotein(a). $J$. Clin. Invest. 92:3029-3037.

16. Borén, J., I. Lee, M.J. Callow, E.M. Rubin, and T.L. Innerarity. 1996. A simple and efficient method for making site-directed mutants, deletions, and fusions of large DNA such as P1 and BAC clones. Genome Res. 6:1123-1130.

17. Yao, Z., B.D. Blackhart, D.F. Johnson, S.M. Taylor, K.W. Haubold, and B.J. McCarthy. 1992. Elimination of apolipoprotein B48 formation in rat hepatoma cell lines transfected with mutant human apolipoprotein B cDNA constructs. J. Biol. Chem. 267:1175-1182.

18. McCormick, S.P.A., M.F. Linton, and S.G. Young. 1994. Expression of P1 DNA in mammalian cells and transgenic mice. Genet. Anal. Tech. Appl. 11: $158-164$.

19. Arnold, K.S., T.L. Innerarity, R.E. Pitas, and R.W. Mahley. 1992. Lipoprotein-receptor interactions. In Lipoprotein Analysis. A Practical Approach C.A. Converse, and E.R. Skinner, editors. Oxford University Press, Oxford. $145-168$.

20. Weisgraber, K.H., T.L. Innerarity, Y.M. Newhouse, S.G. Young, K.S Arnold, R.M. Krauss, G.L. Vega, S.M. Grundy, and R.W. Mahley. 1988. Famil- 
ial defective apolipoprotein B-100: enhanced binding of monoclonal antibody MB47 to abnormal low density lipoproteins. Proc. Natl. Acad. Sci. USA. 85: 9758-9762.

21. Forte, T.M., and R.W. Nordhausen. 1986. Electron microscopy of negatively stained lipoproteins. Methods Enzymol. 128:442-457.

22. Milne, R.W., R. Theolis, Jr., R.B. Verdery, and Y.L. Marcel. 1983. Characterization of monoclonal antibodies against human low density lipoprotein. Arteriosclerosis. 3:23-30.

23. Young, S.G., R.K. Koduri, R.K. Austin, D.J. Bonnet, R.S. Smith, and L.K. Curtiss. 1994. Definition of a nonlinear conformational epitope for the apolipoprotein B-100-specific monoclonal antibody, MB47. J. Lipid Res. 35: 399-407.

24. Farese, R.V., Jr., M.M. Véniant, C.M. Cham, L.M. Flynn, V. Pierotti, J.F. Loring, M. Traber, S. Ruland, R.S. Stokowski, D. Huszar, and S.G. Young. 1996. Phenotypic analysis of mice expressing exclusively apolipoprotein B48 or apolipoprotein B100. Proc. Natl. Acad. Sci. USA. 93:6393-6398.

25. Borchardt, R.A., and R.A. Davis. 1987. Intrahepatic assembly of very low density lipoproteins. Rate of transport out of the endoplasmic reticulum determines rate of secretion. J. Biol. Chem. 262:16394-16402.

26. Chiesa, G., D.F. Johnson, Z. Yao, T.L. Innerarity, R.W. Mahley, S.G. Young, R.H. Hammer, and H.H. Hobbs. 1993. Expression of human apolipoprotein B100 in transgenic mice. Editing of human apolipoprotein B100 mRNA. J. Biol. Chem. 268:23747-23750.

27. Law, A., and J. Scott. 1990. A cross-species comparison of the apolipo- protein B domain that binds to the LDL receptor. J. Lipid Res. 31:1109-1120.

28. Weisgraber, K.H., and S.C. Rall, Jr. 1987. Human apolipoprotein B-100 heparin-binding sites. J. Biol. Chem. 262:11097-11103.

29. Chatterton, J.E., M.L. Phillips, L.K. Curtiss, R. Milne, J.-C. Fruchart, and V.N. Schumaker. 1995. Immunoelectron microscopy of low density lipoproteins yields a ribbon and bow model for the conformation of apolipoprotein B on the lipoprotein surface. J. Lipid Res. 36:2027-2037.

30. Lund-Katz, S., T.L. Innerarity, K.S. Arnold, L.K. Curtiss, and M.C. Phillips. 1991. ${ }^{13} \mathrm{C}$ NMR evidence that substitution of glutamine for arginine 3500 in familial defective apolipoprotein B-100 disrupts the conformation of the receptor-binding domain. J. Biol. Chem. 266:2701-2704.

31. McCormick, S.P.A., J.K. Ng, M. Véniant, J. Borén, V. Pierotti, L.M. Flynn, D.S. Grass, A. Connolly, and S.G. Young. 1996. Transgenic mice that overexpress mouse apolipoprotein B. Evidence that the DNA sequences controlling intestinal expression of the apolipoprotein B gene are distant from the structural gene. J. Biol. Chem. 271:11963-11970.

32. Kleinman, Y., G. Schonfeld, D. Gavish, Y. Oschry, and S. Eisenberg. 1987. Hypolipidemic therapy modulates expression of apolipoprotein B epitopes on low density lipoproteins. Studies in mild to moderate hypertriglyceridemic patients. J. Lipid Res. 28:540-548.

33. Avriam, M., S. Lund-Katz, M.C. Phillips, and A. Chait. 1988. The influence of the triglyceride content of low density lipoprotein on the interaction of apolipoprotein B-100 with cells. J. Biol. Chem. 263:16842-16848. 